\title{
openheart Mobile phone text-messaging interventions aimed to prevent cardiovascular diseases (Text2PreventCVD): systematic review and individual patient data meta-analysis
}

Sheikh Mohammed Shariful Islam (i) , ${ }^{1,2,3}$ Andrew J Farmer, ${ }^{4}$ Kirsten Bobrow, ${ }^{4}$ Ralph Maddison, ${ }^{1}$ Robyn Whittaker, ${ }^{5}$ Leila Anne Pfaeffli Dale, ${ }^{5}$ Andreas Lechner, ${ }^{6}$ Scott Lear, ${ }^{7}$ Zubin Eapen, ${ }^{8}$ Louis Wilhelmus Niessen, ${ }^{9}$ Karla Santo (1) , ${ }^{2,3}$ Sandrine Stepien, ${ }^{2}$ Julie Redfern, ${ }^{2,3}$ Anthony Rodgers, ${ }^{10}$ Clara K Chow, ${ }^{2,3}$ On behalf of Text2PreventCVD Collaboration

\section{- Additional material is published online only. To view, please visit the journal online (http://dx.doi.org/10.1136/ openhrt-2019-001017).}

To cite: Shariful Islam SM, Farmer AJ, Bobrow K, et al. Mobile phone text-messaging interventions aimed to prevent cardiovascular diseases (Text2PreventCVD): systematic review and individual patient data meta-analysis. Open Heart 2019;6:e01017. doi:10.1136/ openhrt-2019-001017

Received 20 January 2019 Revised 18 July 2019 Accepted 11 September 2019

Check for updates

(C) Author(s) (or their employer(s)) 2019. Re-use permitted under CC BY-NC. No commercial re-use. See rights and permissions. Published by BMJ.

For numbered affiliations see end of article.

Correspondence to Professor Clara K Chow; clara. chow@sydney.edu.au

\section{ABSTRACT}

Background A variety of small mobile phone textmessaging interventions have indicated improvement in risk factors for cardiovascular disease (CVD). Yet the extent of this improvement and whether it impacts multiple risk factors together is uncertain. We aimed to conduct a systematic review and individual patient data (IPD) meta-analysis to investigate the effects of text-messaging interventions for CVD prevention.

Methods Electronic databases were searched to identify trials investigating a text-messaging intervention focusing on CVD prevention with the potential to modify at least two CVD risk factors in adults. The main outcome was blood pressure (BP). We conducted standard and IPD meta-analysis on pooled data. We accounted for clustering of patients within studies and the primary analysis used random-effects models. Sensitivity and subgroup analyses were performed.

Results Nine trials were included in the systematic review involving 3779 participants and $5(n=2612)$ contributed data to the IPD meta-analysis. Standard metaanalysis showed that the weighted mean differences are as follows: systolic blood pressure (SBP), $-4.13 \mathrm{~mm} \mathrm{Hg}$ (95\% Cl -11.07 to 2.81, $\mathrm{p}<0.0001)$; diastolic blood pressure (DBP), $-1.11 \mathrm{~mm} \mathrm{Hg}(-1.91$ to $-0.31, \mathrm{p}=0.002)$; and body mass index (BMI), -0.32 ( -0.49 to -0.16 , $\mathrm{p}=0.000)$. In the IPD meta-analysis, the mean difference are as follows: SBP, $-1.3 \mathrm{~mm} \mathrm{Hg}(-5.4$ to 2.7, $\mathrm{p}=0.5236)$; DBP, $-0.8 \mathrm{~mm} \mathrm{Hg}$ ( -2.5 to $1.0, \mathrm{p}=0.3912$ ); and $\mathrm{BMI},-0.2$ $(-0.8$ to $0.4, p=0.5200)$ in the random-effects model. The impact on other risk factors is described, but there were insufficient data to conduct meta-analyses.

Conclusion Mobile phone text-messaging interventions have modest impacts on BP and BMI. Simultaneous but small impacts on multiple risk factors are likely to be clinically relevant and improve outcome, but there are currently insufficient data in pooled analyses to examine the extent to which simultaneous reduction in multiple risk factors occurs.

PROSPERO registration number CRD42016033236.

\section{Key questions}

What is already known about this subject?

- A variety of small mobile phone text-messaging interventions have indicated improvement in risk factors for cardiovascular disease (CVD).

What does this study add?

- This is the first individual patient data meta-analysis showing the impact of text messaging on secondary prevention of CVD.

How might this impact on clinical practice?

- Text messaging is acceptable, generalisable and scalable and has modest impacts on multiple CVD risk factors with public health significance.

\section{INTRODUCTION}

Globally, deaths due to cardiovascular disease (CVD) have steadily increased over the last four decades, ${ }^{1}$ with ischaemic heart diseases remaining as the single largest cause of death. ${ }^{2}$ A large body of evidence has demonstrated that common and modifiable risk factors, including high blood pressure (BP), smoking, high cholesterol, obesity and physical inactivity, contribute substantially to the risk of CVD and premature death. ${ }^{3-8}$ However, identifying low-cost, scalable and effective strategies to target all of these to prevent CVD and recurrent CVD events remains a major challenge.

In recent years, mobile health (mHealth) programmes have emerged as a strategy to support chronic health conditions. Much of the fervour around mHealth has been because of the potential seen to reduce socioeconomic disparity and to deliver a scalable low-cost intervention to a wide population and thus 
alleviate the burden of CVD. ${ }^{9}$ Two systematic reviews on mHealth interventions for secondary prevention of CVD reported that mHealth offers potential for improving CVD secondary prevention. ${ }^{10}{ }^{11}$ The 2018 review $^{7}$ included 9 $(\mathrm{n}=3637)$ studies using text messaging, and the 2016 study $^{8}$ included 28 studies $(\mathrm{n}=3820)$ using mHealth technologies (including text messaging, mobile apps and internet); neither of the reviews performed meta-analysis. The majority of studies showed the effectiveness of mHealth and text messaging in improving behaviours, patient satisfaction and clinical outcomes in patients with CVD. Text messaging and apps showed the highest user adherence and satisfaction compared with internet or continuous monitoring. The 2018 review concluded that text messaging might be a useful tool for secondary prevention of CVD, but conclusions were largely based on qualitative assessment of studies, and the 2016 review concluded that mobile phone features such as text messaging and apps have a strong potential to positively impact the secondary prevention of CVD. They suggested further research was needed to apply rigorous research designs with theory-based interventions, to measure economic benefits, process evaluation and adverse effects of the intervention, and were unable to address the frequency and content of text messaging and long-term impacts of the intervention on clinical outcomes and end points.

The global reach and ubiquity of mHealth interventions suggest they may be useful, but despite almost a decade of research, the evidence base remains limited. In this context, we performed a systematic review and meta-analysis of randomised controlled trials (RCTs) of text-messaging interventions used for the prevention of CVD risk factors to generate robust evidence and to identify researchers active in this field. We established the Text2PreventCVD Trial Collaborators Group, a global network of researchers working on mHealth interventions in CVD prevention and management, with the goals of collaboration to facilitate the next generation of trials. In the current paper, the Text2PreventCVD Collaboration aimed to quantify the extent of improvement in cardiovascular risk factors with mobile phone text-messaging interventions using systematic review and individual participant data (IPD) meta-analysis. Studies included needed to involve interventions that had the potential to impact on at least two measures of cardiovascular risk factors. The primary outcome designated for these analyses was systolic blood pressure (SBP) and the secondary outcomes included diastolic blood pressure (DBP) and body mass index (BMI).

\section{METHODS}

We performed a systematic review and IPD meta-analysis and reported in accordance with the Preferred Reporting Items for Systematic Reviews and Meta-Analyses statement and the Cochrane Collaboration reporting items for systematic reviews and meta-analysis guidelines. ${ }^{12}$ This review is registered in the International Prospective
Register of Systematic Reviews. The study protocol has been published previously. ${ }^{13}$

\section{Search strategy}

We searched MEDLINE, Embase, PsycINFO (Ovid) and the Cochrane Central Register of Controlled Trials for reports published from 1 January 1990 to 1 July 2016 to identify RCTs of text-messaging interventions used for the prevention of CVD risk factors. The following Medical Subject Headings search terms were used: (1) intervention (text messaging, text messages, short message service (SMS), text message mobile phone, cellular phone, texting, SMS); (2) CVD (heart disease, coronary disease, $\mathrm{BP}$, hypertension, lipids, cholesterol, cardiovascular risk factors, myocardial infarction, vascular, diabetes and obesity) and (3) study design (RCT). We also searched for ongoing, recently completed and unpublished clinical trials meeting the inclusion criteria described earlier from different trial registers and reference lists of selected studies, and reviewed grey literature for any other relevant studies. Consultation and contacts with experts in the field were made to help identify relevant studies.

\section{Study eligibility}

Full details of the methods used have been reported previously. ${ }^{13}$ In brief, studies were eligible if they were RCTs of a text-messaging intervention focused on CVD prevention (primary or secondary) with at least two behaviour change strategies, for example, physical activity and diet or BP lowering, smoking and cholesterol lowering ${ }^{14}$; study duration was at least 6 months and $70 \%$ completed the follow-up of participants; study participants were adults, the sample size was larger than 30 people, and the control group received standard care. Reports published in any language were considered.

\section{Study selection and data extraction}

Two researchers (SMSI and KS) independently screened titles and abstracts to identify studies meeting the inclusion criteria described previously. One researcher (SMSI) extracted and tabulated all relevant data in a predefined data extraction sheet and a second researcher (KS) checked it for accuracy. Extracted data included patient's baseline characteristics; the type and duration of the intervention; changes in SBP and DBP, BMI, weight and lipids; and any possible adverse outcome reported. Disagreement was resolved by consensus or in consultation with a third reviewer (CKC). Corresponding authors of the selected studies were invited to join the Text2PreventCVD Collaboration and to contribute data to the IPD meta-analysis.

\section{Outcomes}

The primary outcome was the difference between intervention and control groups in SBP at 6 months. However, as two studies ${ }^{15} 16$ did not have complete SBP data at 6 months' follow-up, we performed the analysis based on end of follow-up data. We also sought to examine other measures of cardiovascular risk, including low-density lipoprotein (LDL) cholesterol, smoking, diet, quality of 
life and physical activity. However, due to insufficient data availability, we were only able to conduct meta-analysis to analyse diastolic BP and BMI as secondary outcomes at the end of the follow-up period.

\section{Assessment of study quality}

Study quality was assessed using the Cochrane collaboration risk of bias assessment tool, ${ }^{17}$ based on random sequence generation, allocation concealment, blinding of participants and personnel, blinding of outcome assessment, incomplete outcome data, selective reporting and other bias.

\section{Statistical analysis}

Analyses were conducted in accordance with contemporary recommendations for IPD meta-analyses. ${ }^{18-21}$ Primary and secondary outcome analyses were performed on the combined dataset using preferred one-stage IPD meta-analyses (ie, individual patient data (IPD) were pooled and then models run on the combined dataset). ${ }^{202}$ The primary analyses consisted of a linear mixed model with the end-of-trial value as the outcome, the baseline value and the treatment arm as random effects, and a random trial intercept and random trial-bytreatment interaction. An interim meta-analysis (standard meta-analysis) of the systematic review was performed using both fixed and random-effects models to compare the results with the IPD meta-analysis. Sensitivity analyses included two-stage approaches using both random and fixed-effects meta-analyses, analysis of 6-month follow-up values as the outcome and excluding data from the heart exercise and remote technologies (HEART) study, which measured BP in the context of participants doing exercise. Subgroup analyses were performed using the one-stage IPD and fitting a subgroup and subgroup by treatment interaction fixed effect into the model so heterogeneity between subgroup categories (education, age and gender) could be assessed. All data analyses were carried out using STATA V.12 and SAS V.9.2.

\section{RESULTS}

\section{Study characteristics}

Out of a total of 1210 identified citations, nine trials consisting of 3779 participants met the inclusion criteria of our systematic search (figure 1). We contacted all nine study groups and five agreed to contribute data to the IPD meta-analysis described further. Characteristics of the nine trials are shown in table 1 . The median sample size was 236 (range 123-1372) and the median intervention period was 6 months (range 6-24 months). The mean age of the participants was $54.1 \pm 5.5$ years and $37.5 \%$ were women. The gender spread across the studies was broad from one study of $100 \% \mathrm{men}^{23}$ to one with $<30 \% .{ }^{15}$ The trials included participants with a range of levels of absolute cardiovascular risk from primary prevention to secondary prevention populations. Of the nine trials included, one trial included 'normal' participants not on drug therapy, ${ }^{23}$ with the others including

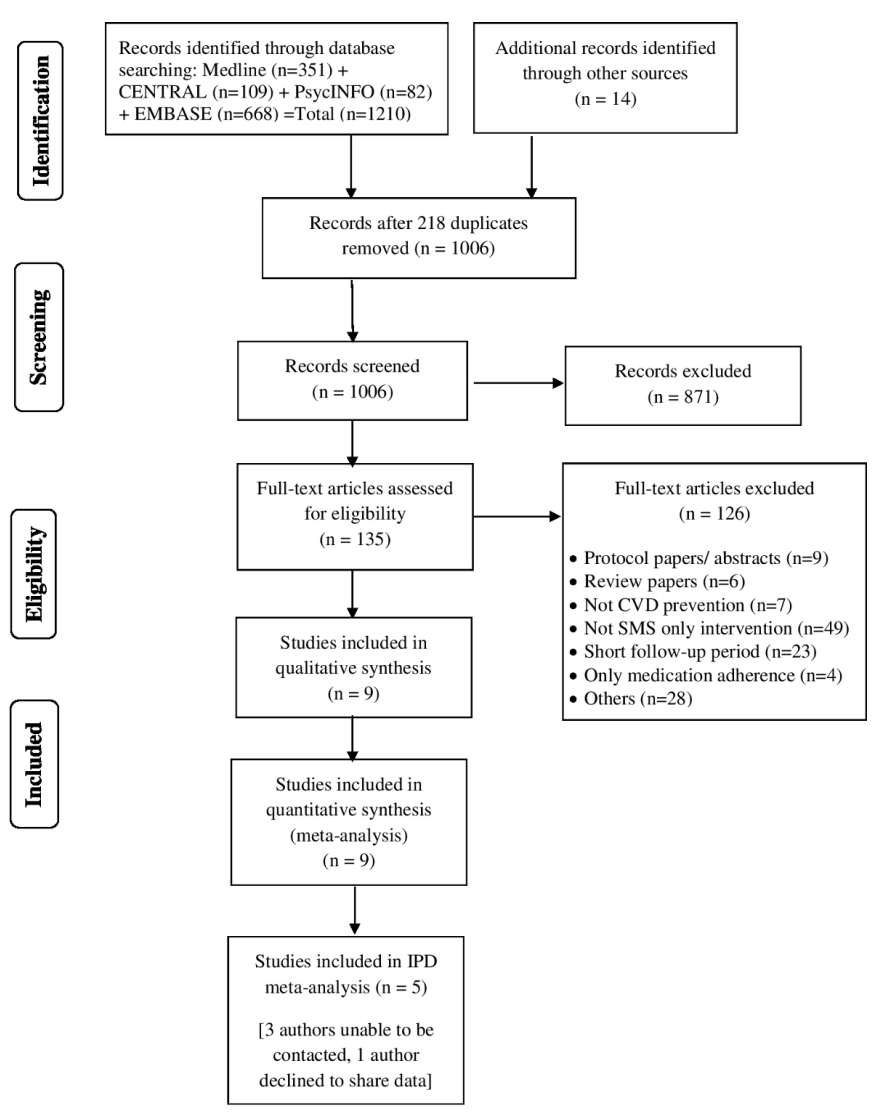

Figure 1 Study selection process. CENTRAL, Cochrane Central Register of Controlled Trials. CVD, cardiovascular disease; IPD, individual patient data; SMS, short message service.

patient populations selected on the basis of having a cardiovascular risk factor or CVD; for example, two studies included participants with high $\mathrm{BP},{ }^{15}{ }^{16}$ three with coronary heart diseases, ${ }^{24-26}$ two with diabetes, ${ }^{27} 28$ one included only working men with no diabetes ${ }^{23}$ and one participant taking medication for either BP or lipid lowering. ${ }^{29}$ Five studies recruited participants from hospital settings, ${ }^{1624-26} 28$ two from primary healthcare settings, ${ }^{1529}$ one from a hospital emergency department ${ }^{27}$ and one in an industrial area work setting. ${ }^{23}$ The duration of the text-messaging intervention was 6 months for seven studies, ${ }^{24-29} 12$ months for one ${ }^{15}$ and 24 months for another. ${ }^{23}$

\section{Text message intervention characteristics}

There was considerable variation in the characteristics of the individual text-message interventions (see online supplementary table S1). Five studies provided text messages as a stand-alone intervention. Four studies provided additional support to participants in the form of telephone support ${ }^{1629}$ and via secure website. ${ }^{2526}$ Message frequency varied from one message per week up to two messages per day. Four studies sent text messages at a fixed predetermined frequency. ${ }^{24}{ }^{27-29}$ Most studies used unidirectional text messaging and three used interactive text messaging. ${ }^{152529}$ Six studies used personalised or semipersonalised text messages, while three studies used 


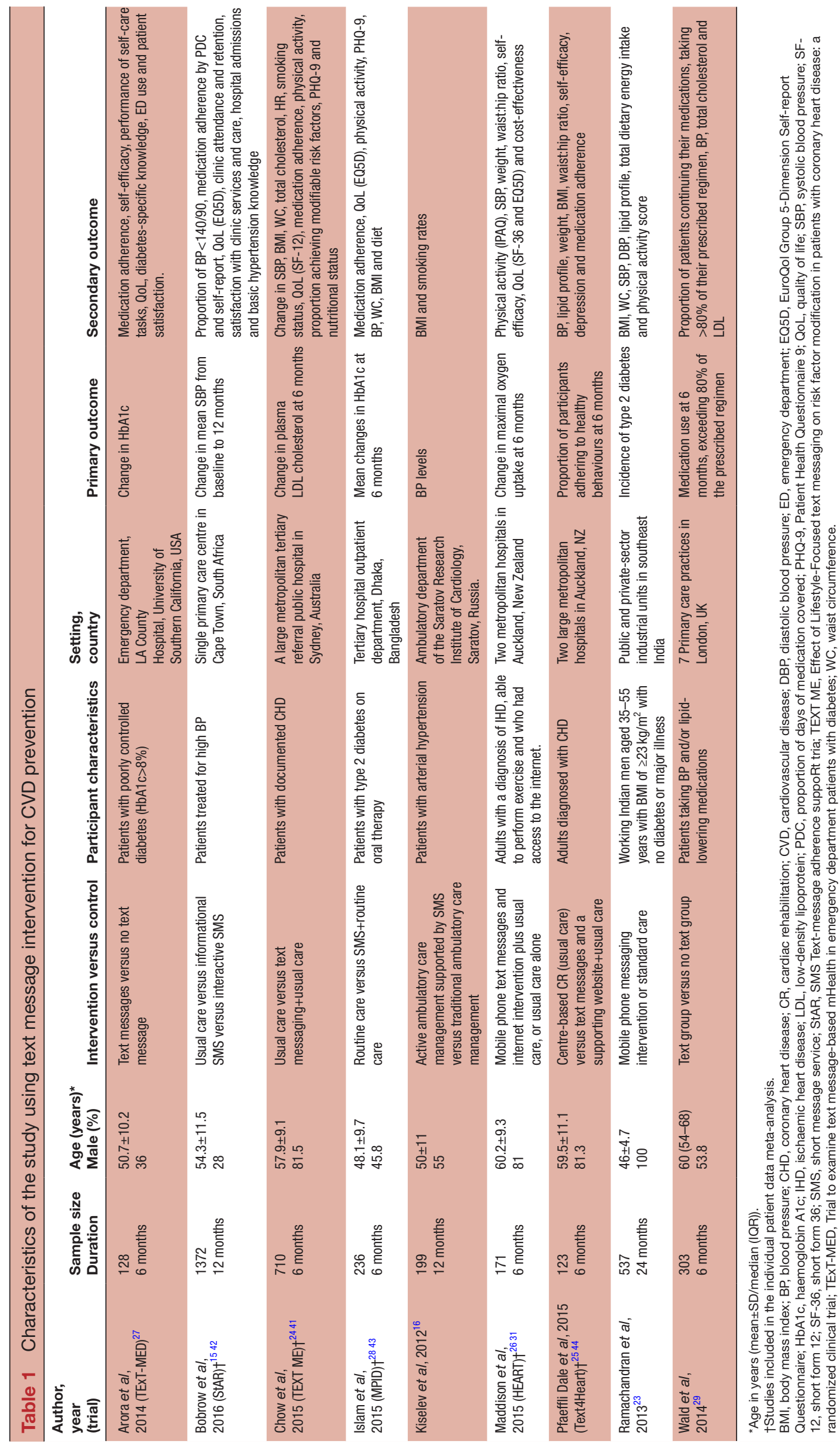




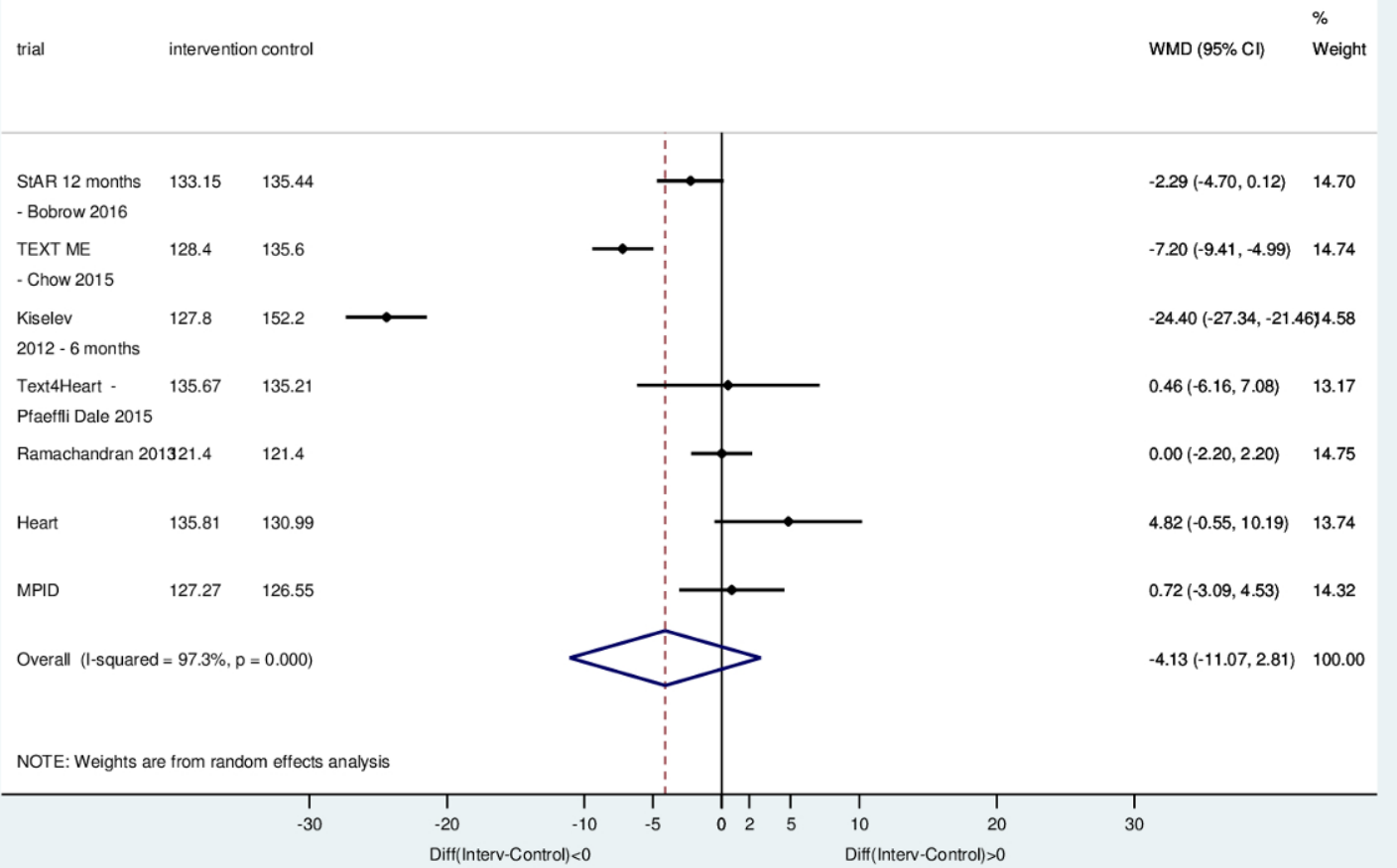

Figure 2 Standard meta-analysis at end follow-up on systolic blood pressure results (random-effects model). MPID, mobile phone intervention for diabetes; WMD, weighted mean difference.

generalised messages. ${ }^{16} 2728$ Message content was based on clinical guidelines (five studies), expert opinion (two studies) and feedback from formative qualitative work (three studies). The number of behaviour change strategies used ranged from 2 to 17 . Seven studies reported patient feedback on the text messaging. ${ }^{1623-29}$

\section{Standard meta-analysis of the intervention efficacy}

Of the 3779 participants, 295 did not have complete data and were excluded from the analysis. In the pooled analysis of 3348 patients, using a random-effects model, text message intervention significantly reduced SBP at the end of follow-up (weighted mean difference $-4.13 \mathrm{~mm} \mathrm{Hg}$ (95\% CI -11.07 to $-2.81, \mathrm{p}=<0.0001$ ) (figure 2 ). There was high heterogeneity $\left(\mathrm{I}^{2} 97.3 \%\right)$ across the clinical trials due to one study with a large effect size. ${ }^{16}$ The weighted mean difference in DBP was $-1.11 \mathrm{~mm} \mathrm{Hg}(95 \%$ CI -1.91 to $\left.-0.31, \mathrm{p}=0.002, \mathrm{I}^{2} 77.2 \%\right)$ and BMI was $-0.32(95 \% \mathrm{CI}$ -0.49 to $\left.-0.158,<0.0001, \mathrm{I}^{2} 91.5 \%\right)$.

\section{IPD meta-analysis of the intervention efficacy}

The IPD meta-analysis involved five studies with 1976 participants from Australia, Bangladesh, New Zealand and South Africa (table 2). The mean difference in SBP at the end of the follow-up period was $-1.3 \mathrm{~mm} \mathrm{Hg}(95 \% \mathrm{CI}-5.4$ to 2.7, $\left.\mathrm{p}=0.5236, \mathrm{I}^{2} 89.0 \%\right)$ in the random-effects model. The mean differences in DBP and BMI were $-0.8 \mathrm{~mm} \mathrm{Hg}(95 \%$ CI -2.5 to $\left.1.0, \mathrm{p}=0.3912, \mathrm{I}^{2} 77.2 \%\right)$ and $-0.2 \mathrm{~mm} \mathrm{Hg}(95 \%$ CI -0.8 to $0.4, \mathrm{p}=0.5200, \mathrm{I}^{2} 91.5 \%$ ), respectively, at the end of the follow-up using the random-effects model (table 3 ).

\section{Other CVD risk factors}

Three studies ${ }^{2527} 28$ included in the standard meta-analysis reported effects of text messaging on medication adherence using Morisky's eight-item medication adherence scale (MMAS-8). ${ }^{30}$ These studies showed improvement in the MMAS-8 score to a varying degree 1.1 (95\% CI 0.1 to 2.1$),-0.11(95 \% \mathrm{CI}-0.49$ to 0.26$)$ and 0.58 (95\% CI 0.19 to 0.97$)$. Three studies measured changes in LDL cholesterol, but data were presented in reports for only two studies $-0.11 \mathrm{mg} / \mathrm{dL}(95 \% \mathrm{CI}-0.49 \text { to } 0.26, \mathrm{p}=0.04)^{24}$ and $-0.25 \mathrm{mg} / \mathrm{dL}(95 \% \mathrm{CI}-0.49$ to $0.01, \mathrm{p}=0.053){ }^{25}$ Two studies reported changes in glycated haemoglobin with a mean difference of $-0.45 \%(\mathrm{p}=0.230)^{27}$ and $-0.64 \%(-0.95$ to -0.33$){ }^{28}$

With respect to whether interventions impacted on multiple cardiovascular risk factors, four studies ${ }^{15232428}$ showed statistically significant reductions in two or more cardiovascular risk factors, while two studies ${ }^{27}{ }^{31}$ reported improvements (not statistically significant) in two or more cardiovascular risk factors at the end of the study (see online supplementary table S5). One study reported concurrent reductions in LDL cholesterol, BP, BMI, physical activity and smoking, with $28.9 \%$ achieving at least four guideline levels of cardiovascular risk factors in the intervention group, compared with $10.3 \%$ in the control group and $4.7 \%$ achieving all five guideline levels in the intervention group, compared with $1.8 \%$ in the control group. ${ }^{24}$

\section{Sensitivity analysis}

A sensitivity analysis was conducted in which data from the HEART trial ${ }^{26}$ were excluded, mainly because BP 


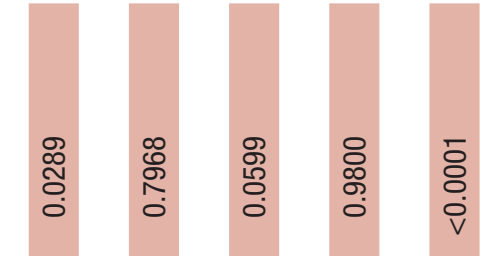

(1)

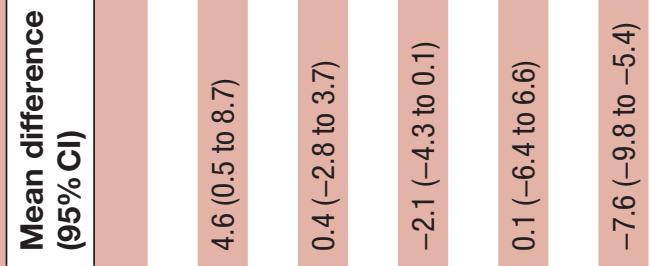

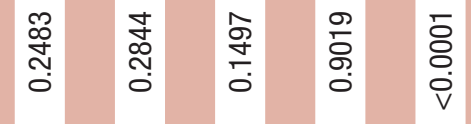

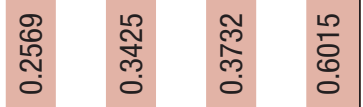

o

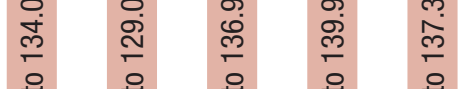

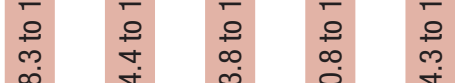

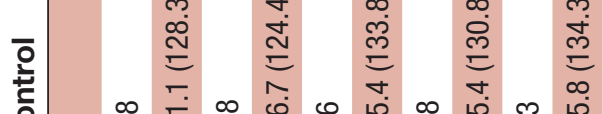

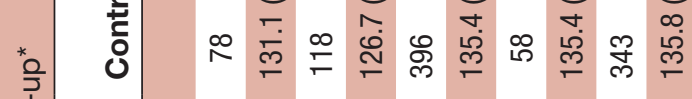

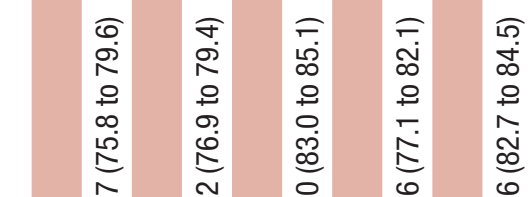

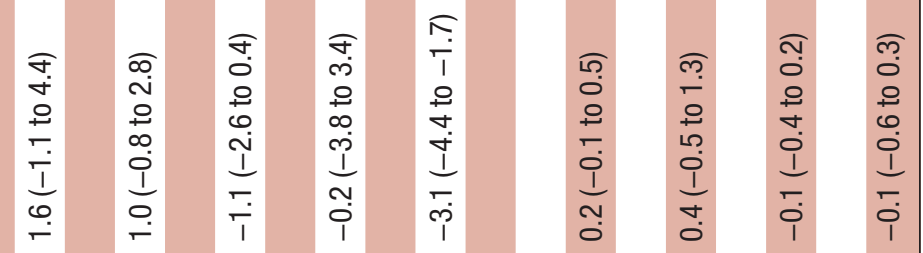

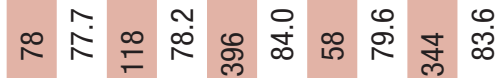

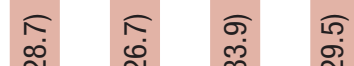

$\frac{\sum^{3}}{\underline{\underline{O}}}$

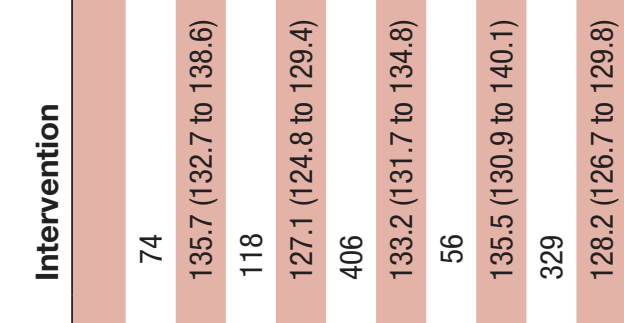

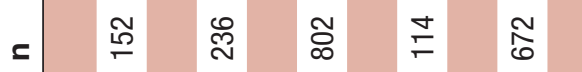

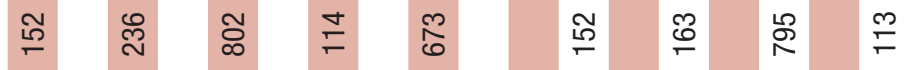

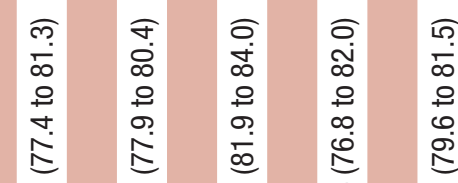

i $\frac{1}{2}$ in

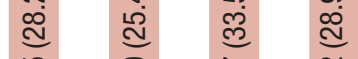

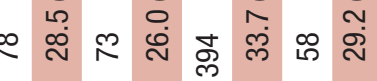

雨

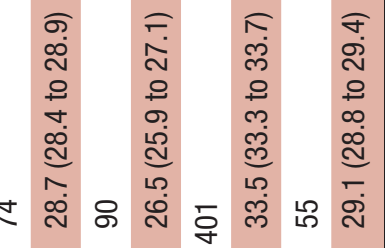

然

हैं

岳

$\frac{\frac{\infty}{\infty}}{\frac{\pi}{\sigma}}$

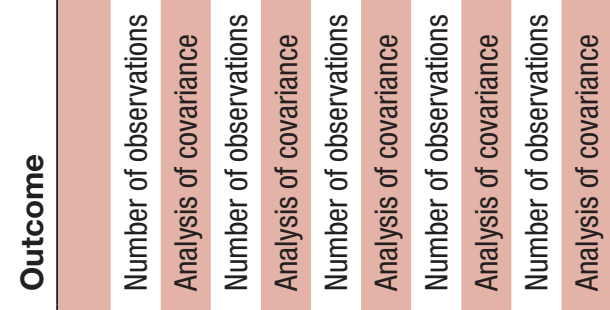

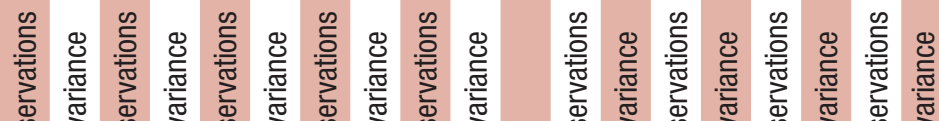

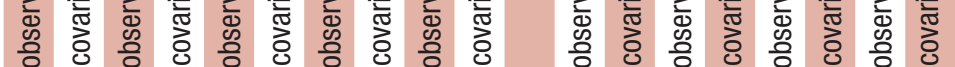
¿

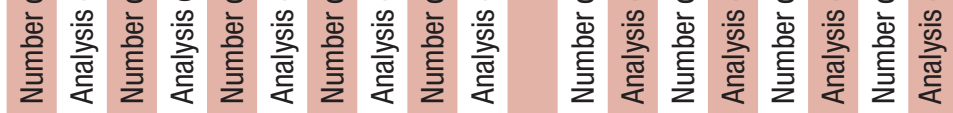

$\frac{\substack{0 \\ \frac{1}{\pi}}}{\frac{0}{0}}$

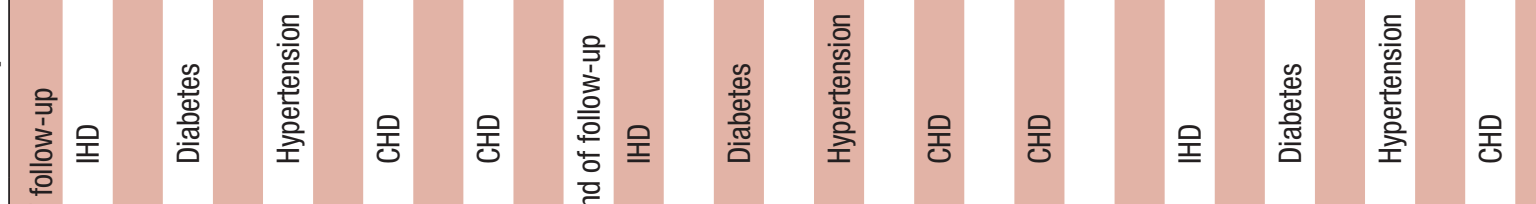

$\frac{\text { Do }}{\sum_{\bar{O}}^{\circ}}$

$\stackrel{\frac{1}{ \pm}}{\leftrightarrows}$

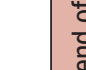

등

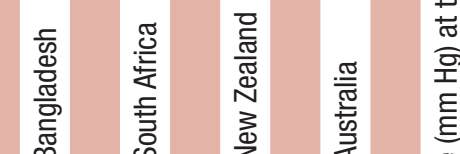

하

竞

일

ङ

胥 के

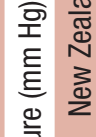

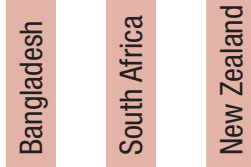

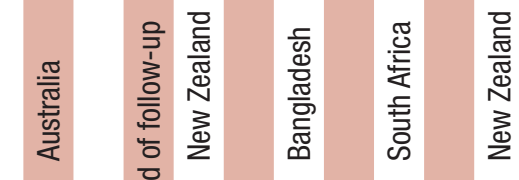

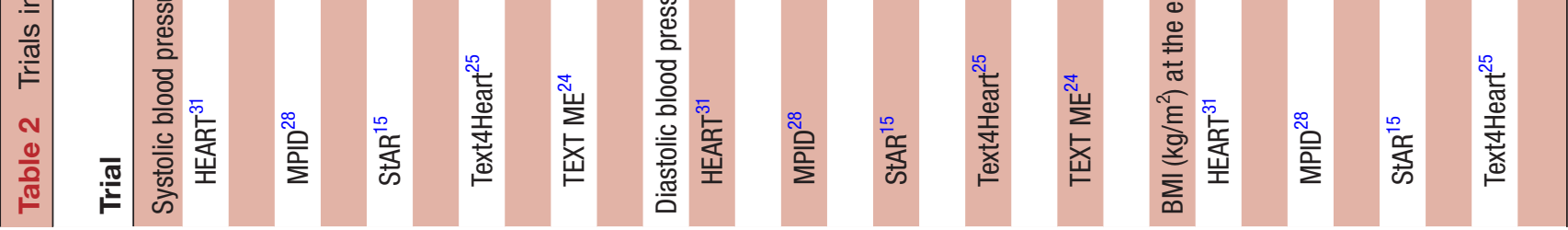

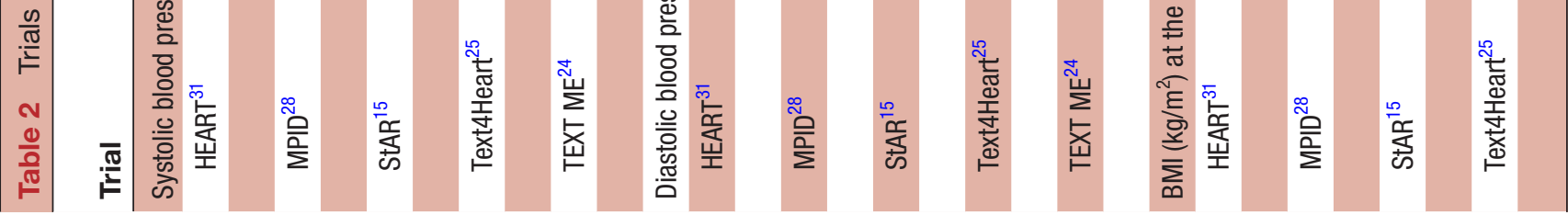




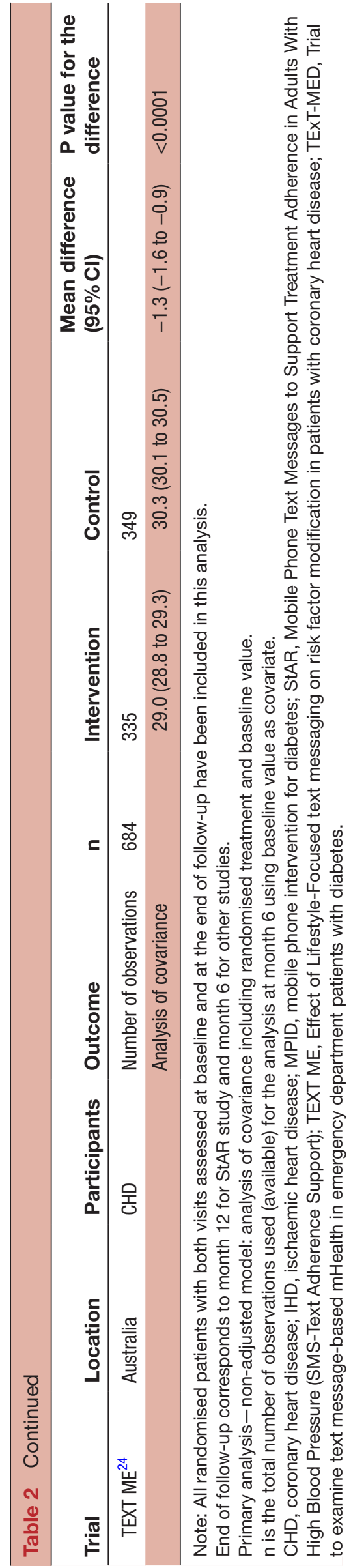

was measured in the context of cardiopulmonary exercise testing. As a result, reductions were found for SBP $-3.7 \mathrm{~mm} \mathrm{Hg}(95 \% \mathrm{CI}-5.1$ to -2.3$)$, DBP $-1.6 \mathrm{~mm} \mathrm{Hg}$ $(95 \%$ CI -2.5 to -0.7$)$ and BMI -0.5 (95\% CI -0.7 to -0.3$)$ (see online supplementary table S2).

\section{Effects stratified by subgroup analysis}

To further assess the impact of text messages, we performed an analysis based on participant characteristics. The moderators assessed were education $(<12$ years vs $\geq 12$ years), age ( $<60$ years vs $\geq 60$ year) and gender (male vs female). There was no significant variation in the effectiveness of text message intervention by strata (see online supplementary table S3).

\section{Study quality}

We assessed the study quality as per Cochrane guidelines ${ }^{12}$ (see online supplementary table S4). All studies described a randomisation sequence generation technique that was at low risk of bias except one that had an unclear risk of bias. ${ }^{16}$ Three studies did not report allocation concealment and were categorised as unclear allocation concealment. ${ }^{1623} 27$ Blinding of study participants was not possible due to the nature of intervention for any of the studies. Blinding of outcome assessments was not clearly described in four studies. ${ }^{162527} 29$ Six studies were reported to have low incomplete outcome data, ${ }^{1523-27} 29$ one with unclear outcome data ${ }^{28}$ and one with high incomplete outcome data. ${ }^{16}$ All studies scored low in selective reporting and other bias, while only one study had unclear other bias. ${ }^{16}$ Overall, 7 (78\%) studies were thought to be of high quality with a maximum of one unclear assessment. ${ }^{1523-2628} 29$ Publication bias could not be assessed as the number of trials was few.

\section{Text message acceptability}

Participant feedback on text-message acceptability was reported in seven studies ${ }^{1623-28}$ (see online supplementary table S2). Most studies reported moderate to high levels of satisfaction with the text-messaging programme. Participants acknowledged text-message support as a useful and expressed desire to programme continuation. In one study, ${ }^{28}{ }^{32}$ participants were willing to pay a small fee to receive the text-messaging programme. In one study, a small fraction (3\%) of the participants felt that the messages were disturbing. ${ }^{23}$

\section{DISCUSSION}

The main findings of this paper is that mobile phone text-messaging interventions have modest impacts on objective measures of cardiovascular risk factors of BP and BMI in analyses of pooled data using standard and IPD meta-analyses. Modest impacts on cardiovascular risk factors could have clinical and/or public health significance if they could impact on multiple risk factors simultaneously and/or do this at a very low cost, but the current data are not conclusive on these points. The pooled data are from a number of different regions, and the high 
Table 3 Individual patient data meta-analysis at the end of follow-up*

\begin{tabular}{|c|c|c|c|c|c|}
\hline Outcome & Model & Intervention & Control & $\begin{array}{l}\text { Mean difference } \\
(95 \% \mathrm{Cl})\end{array}$ & $\begin{array}{l}\text { P value for the } \\
\text { difference }\end{array}$ \\
\hline SBP & Random-effects model (1a) & 132.1 (128.7 to 135.5$)$ & 133.4 (130.0 to 136.8$)$ & $-1.3(-5.4$ to 2.7$)$ & 0.5236 \\
\hline \multirow[t]{2}{*}{ DBP } & Random-effects model (1a) & 80.9 (79.4 to 82.4) & 81.7 (80.2 to 83.2) & $-0.8(-2.5$ to 1.0$)$ & 0.3912 \\
\hline & Fixed-effects model (2) & 80.6 (79.8 to 81.3 ) & 81.9 (81.2 to 82.6$)$ & $-1.3(-2.2$ to -0.5$)$ & 0.0018 \\
\hline
\end{tabular}

Note: All randomised patients with both visits assessed at baseline and at month 6 have been included in this analysis.

Primary analysis - analysis of covariance, including randomised treatment and baseline value as fixed effect: model 1: (a) includes trial random effect and random treatment by trial interaction, (b) includes trial fixed effect and random treatment by trial interaction when model 1 a estimates trial random effect to zero; model 2: only fixed effects, including trial effect; model 3 : sensitivity analysis pooling the estimates from the five trials using a standard meta-analysis checking for data heterogeneity between trials.

*For the StAR study, 12 month data was used for follow-up. For all other studies, 6 month data was used for follow-up. BMI, body mass index; DBP, diastolic blood pressure; SBP, systolic blood pressure.

acceptability of texting interventions reported by most studies in our review suggests generalisability of findings and potential for widespread implementation, but there is still relatively few large-scale or multicentre evaluations.

Efficient, simultaneous risk factor reduction is important in the prevention of CVD as risk reduction is additive across single risk factors; for example, BP lowering plus lipid lowering is more effective at reducing risk than either alone. The reasons that the data are still inconclusive are likely because of the small number of individual participants in trials, the heterogeneity of the study population (including primary and secondary prevention populations), the heterogeneity of the interventions and the likely small individual impact of the interventions themselves.

Mobile phone text messaging might be a low-cost intervention, but data on cost-effectiveness are still limited. One of the studies included had a published cost-effectiveness study that modelled implementation of the text-message intervention to a target population of 50000 patients with coronary heart disease; the intervention was estimated to lead to 563 fewer myocardial infarctions, 361 fewer strokes and 1143 additional quality adjusted life years, and was associated with an overall saving of $\$ 10.56$ million for the health system over the patients' lifetimes. ${ }^{33}$

There have been some previous systematic reviews in mHealth interventions for CVD secondary prevention. One recent systematic review of mobile phone interventions for secondary prevention of CVD reported that text messaging was effective in improving clinical outcomes better than smartphone-based interventions. ${ }^{11}$ The study discussed that incorporating principles of behavioural change may help promote and sustain healthy lifestyle behaviours in patients with CVD, resulting in better outcomes. ${ }^{11}$ Another systematic review of mobile phone text messaging for improving secondary prevention in CVD suggested that text messaging might be beneficial for secondary prevention of CVD but failed to draw reliable conclusions. ${ }^{10} \mathrm{~A}$ 2017 systematic review identified six studies using text messaging for hypertension management but did not perform meta-analysis due to variation in the studies. ${ }^{34}$ The study concluded that text messaging had strong potential for innovation in hypertension management, especially in minority groups and those with low access to healthcare services. All these systematic reviews suggested that text messaging might have a role in secondary prevention of CVD but could not quantify effectiveness due to a lack of meta-analysis. Our study is therefore unique as it used IPD as well as meta-analysis.

The main mechanism by which texting intervention appear to work is via behavioural change. All trials included in our review incorporated two or more behaviour change theories. Behaviour change interventions and techniques, including motivational interviewing, ${ }^{35}$ increasing patient motivation and self-efficacy, ${ }^{36}$ goal setting combined with self-monitoring of behaviour, ${ }^{37}$ supporting medical adherence and multimodal behavioural interventions, have been highlighted as effective in various CVD prevention programmes. ${ }^{38} 39$ Text messaging offers confidential and unobtrusive support, which is an advantage over other interventions.

\section{Strengths and limitations}

The primary limitation of this meta-analysis was the differences in participants' characteristics, risk factor measurements and primary outcomes of interest across studies. A potential limitation is the small number of trials and a highly significant study included in the meta-analysis, ${ }^{16}$ potential publication bias and influence of heterogeneity is possible. Of the nine trials included in the standard meta-analysis, we were only able to obtain full datasets from five trials. Also, we calculated the effect estimate from available data from published studies, and it is thus possible that bias can be introduced from differential loss to follow-up. Therefore, the results of this IPD meta-analysis cannot be generalised across different populations and should be interpreted with caution. Finally, the meta-analysis is not a 
mechanistic study; thus, we cannot determine whether the benefit associated with the use of text messaging was attributable to BP lowering or to other mechanisms.

The primary strength of this meta-analysis was its systematic search to identify potential studies and the inclusion of only RCTs, which are less subject to bias and confounding than observational studies. The IPD analysis approach used in this study has advantages over a meta-analysis because the outcomes can be matched between studies and all studies are then analysed in the same prespecified statistical model. The use of data from many studies with different inclusion criteria should make the findings more generalisable, and the robustness of the main conclusions to subsidiary methods of analysis further supports the conclusion drawn.

\section{Future research}

There are still a number of unanswered questions that we were unable to adequately address, for example, whether different characteristics of the texting interventions influenced outcomes (ie, number of behaviour change techniques used, unidirectional vs bidirectional, high vs low frequency, personalised vs non-personalised). How can effects be maintained into the longer term and how are interventions best scaled up to populations? Future studies should address these questions, as well as the potential of these interventions in varied socioeconomic groups, age groups and cultures. Variations in effectiveness of behavioural interventions have been shown by gender. ${ }^{37} 40$

In addition, substantial variability exists with regard to the definitions of outcome measures for the different cardiovascular risk factors across studies. A standardised method for measuring the different outcomes in CVD prevention is warranted to improve comparability of outcome measures across studies for a more rigorous and reliable evaluation of the effectiveness of mHealth interventions for CVD.

\section{CONCLUSION}

Mobile phone text-messaging interventions have modest impacts on objective measures of BP and BMI. Evidence is suggestive, but inconclusive, that texting is acceptable, generalisable and scalable, and their modest impacts on cardiovascular risk factors may apply to multiple cardiovascular risk factors and hence make this a mHealth solution with public health significance.

\section{Author affiliations}

${ }^{1}$ Institute for Physical Activity and Nutrition, Deakin University, Geelong, Victoria, Australia

${ }^{2}$ Cardiovascular Division, The George Institute for Global Health, Sydney, New South Wales, Australia

${ }^{3}$ Faculty of Medicine and Health, The University of Sydney, Sydney, New South Wales, Australia

${ }^{4}$ Nuffield Department of Primary Care Health Science, University of Oxford, Oxford, United Kingdom

${ }^{5}$ National Institute for Health Innovation, The University of Auckland, Auckland, New Zealand

${ }^{6}$ Medizinische Klinik und Poliklinik IV, Ludwig-Maximillian Universität, Munich, Germany

${ }^{7}$ St. Paul's Hospital, Vancouver, British Columbia, Canada
${ }^{8}$ Department of Medicine, Duke University, Durham, North California, United States ${ }^{9}$ Faculty of International Public Health and Clinical Sciences, Liverpool School of Tropical Medicine, Liverpool, United Kingdom

${ }^{10}$ Professorial Unit, The George Institute for Global Health, Newtown, New South Wales, Australia

\section{Twitter Sheikh Mohammed Shariful Islam @drsislam}

Acknowledgements The authors acknowledge the role of Roderick Dyson, academic librarian at The University of Sydney, who helped to design the search strategy and facilitated study identification. The authors also thank the George Institute for organising two annual meetings of the Text2PreventCVD investigators in 2015 and 2016 in Sydney, Australia.

Collaborators Text2PreventCVD Trial Collaborator Group: current membership of the Group: study institutes and investigators: 1. Cardiovascular Division, The George Institute for Global Health, Sydney, Australia (Secretariat) and University of Sydney, Sydney, Australia: Clara Chow, Julie Redfern, Anthony Rodgers, Shariful Islam and Aravinda Thiagalingam (TEXT ME ${ }^{41}$ ). 2. University of Oxford, Nuffield Department of Primary Care Health Sciences, Oxford, UK/Chronic Disease Initiative for Africa, Division of Diabetes and Endocrinology, Department of Medicine, University of Cape Town and Groote Schuur Hospital, Cape Town, South Africa South Africa: Kirsty Bobrow and Andrew Farmer $\left(S_{A} A R^{42}\right)$. 3. CenterCentre for International Health, Ludwig-Maximilians University, Munich, Germany/ Liverpool School of Tropical Medicine, UK, and Non-Communicable Diseases Initiative, International Center for Diarrhoeal Diseases, Research, Bangladesh (ICDDR,B), Dhaka, Bangladesh: Andreas Lechner, Louis Niessen and Shariful Islam $\left(\mathrm{MPID}^{43}\right)$. 4. National Institute for Health Innovation, The University of Auckland, New Zealand: Ralph Maddison, Robyn Whittaker and Leila Pfaeffli Dale (HEART ${ }^{26}$ and Text4Heart ${ }^{44}$ ). 5. Deakin University, Melbourne, Australia: Ralph Maddision and Shariful Islam. 6. Simon Fraser University and St. Paul's Hospital, Vancouver, BC, Canada: Scott Lear. 7. Duke University, USA: Zubin Eapen.

Contributors SMSI contributed to conception, drafted the protocol and data collection forms, conducted search, data abstraction and data checking as first reviewer, led the statistical analysis, and drafted and revised the paper. CKC contributed to the conception, drafted the protocol, data collection forms, supervised research staff working on the project, contributed to the review of the data analysis and the manuscript. KS conducted search, data abstraction and data checking as second reviewer. SS guided on the statistical analysis, contributed to the review of the data analysis and review of the manuscript. All authors reviewed the draft paper and contributed scientifically to improve the manuscript. SMSI and $\mathrm{CKC}$ are the guarantors.

Funding SMSI was funded by a senior research fellowship from the Institute for Physical Activity and Nutrition, Deakin University, and was a recipient of The George Institute for Global Health postdoctorate fellowship and early career transition grant from the High Blood Pressure Research Council of Australia. AF is a National Institute for Health Research (NIHR) senior investigator and received support from NIHR Oxford Biomedical Research Centre. JR was funded by a Career Development and Future Leader Fellowship cofunded by the National Health and Medical Research Council and the National Heart Foundation (APP1061793). AR has a NHMRC Principal Fellowship. CKC is funded by Career Development Fellowship cofunded by the National Health and Medical Research Council and the National Heart Foundation and the Sydney Medical Foundation Chapman Fellowship (1033478). KS is funded by a University of Sydney International Postgraduate Research Scholarship.

Competing interests None declared.

Patient consent for publication Not required.

Provenance and peer review Not commissioned; internally peer reviewed.

Data availability statement Data are available upon reasonable request.

Author note All authors satisfied the four ICMJE Recommendations 2013 criteria for authorship.

Open access This is an open access article distributed in accordance with the Creative Commons Attribution Non Commercial (CC BY-NC 4.0) license, which permits others to distribute, remix, adapt, build upon this work non-commercially, and license their derivative works on different terms, provided the original work is properly cited, appropriate credit is given, any changes made indicated, and the use is non-commercial. See: http://creativecommons.org/licenses/by-nc/4.0/.

ORCID iDs

Sheikh Mohammed Shariful Islam http://orcid.org/0000-0001-7926-9368 
Karla Santo http://orcid.org/0000-0001-9047-8865

\section{REFERENCES}

1 Naghavi M, Abajobir AA, Abbafati C, et al. Global, regional, and national age-sex specific mortality for 264 causes of death, 19802016: a systematic analysis for the global burden of disease study 2016. Lancet 2017;390:1151-210.

2 Finegold JA, Asaria P, Francis DP. Mortality from ischaemic heart disease by country, region, and age: statistics from World health organisation and United nations. Int J Cardiol 2013;168:934-45.

3 Blair SN, Kohl HW, Paffenbarger RS, et al. Physical fitness and all-cause mortality. A prospective study of healthy men and women. JAMA 1989;262:2395-401.

$4 \mathrm{Hu}$ FB, Willett WC, Li T, et al. Adiposity as compared with physical activity in predicting mortality among women. $N$ Engl $J$ Med 2004;351:2694-703

5 Wessel TR, Arant CB, Olson MB, et al. Relationship of physical fitness vs body mass index with coronary artery disease and cardiovascular events in women. JAMA 2004;292:1179-87.

6 Forouzanfar MH, Liu P, Roth GA, et al. Global burden of hypertension and systolic blood pressure of at least 110 to $115 \mathrm{MM} \mathrm{Hg}, 1990-$ 2015. JAMA 2017;317:165-82.

7 Gakidou E, Afshin A, Abajobir AA, et al. Global, regional, and national comparative risk assessment of 84 behavioural, environmental and occupational, and metabolic risks or clusters of risks, 1990-2016: a systematic analysis for the global burden of disease study 2016 . Lancet 2017;390:1345-422.

8 Ezzati M, Lopez AD. Estimates of global mortality attributable to smoking in 2000. Lancet 2003;362:847-52.

9 Chow CK, Ariyarathna N, Islam SMS, et al. mHealth in cardiovascular health care. Heart Lung Circ 2016;25:802-7.

10 Unal E, Giakoumidakis K, Khan E, et al. Mobile phone text messaging for improving secondary prevention in cardiovascular diseases: a systematic review. Heart Lung 2018;47:351-9.

11 Park LG, Beatty A, Stafford Z, et al. Mobile phone interventions for the secondary prevention of cardiovascular disease. Prog Cardiovasc Dis 2016;58:639-50.

12 Higgins JP, Green S. Cochrane Handbook for systematic reviews of interventions. John Wiley \& Sons, 2011.

13 Chow CK, Islam SMS, Farmer A, et al. Text2PreventCVD: protocol for a systematic review and individual participant data metaanalysis of text message-based interventions for the prevention of cardiovascular diseases. BMJ Open 2016;6:e012723.

14 Michie S, Richardson M, Johnston M, et al. The behavior change technique taxonomy (V1) of 93 hierarchically clustered techniques: building an international consensus for the reporting of behavior change interventions. Ann Behav Med 2013;46:81-95.

15 Bobrow K, Farmer AJ, Springer D, et al. Mobile Phone Text Messages to Support Treatment Adherence in Adults With High Blood Pressure (SMS-Text Adherence Support [StAR]): A SingleBlind, Randomized Trial. Circulation 2016;133:592-600.

16 Kiselev AR, Gridnev VI, Shvartz VA, et al. Active ambulatory care management supported by short message services and mobile phone technology in patients with arterial hypertension. J Am Soc Hypertens 2012;6:346-55.

17 Higgins JPT, Altman DG, Gøtzsche PC, et al. The Cochrane collaboration's tool for assessing risk of bias in randomised trials. BMJ 2011;343:d5928.

18 Simmonds MC, Higgins JPT, Stewart LA, et al. Meta-analysis of individual patient data from randomized trials: a review of methods used in practice. Clin Trials 2005;2:209-17.

19 Stewart GB, Altman DG, Askie LM, et al. Statistical analysis of individual participant data meta-analyses: a comparison of methods and recommendations for practice. PLoS One 2012;7:e46042.

20 Webster R, Patel A, Selak V, et al. Effectiveness of fixed dose combination medication ('polypills') compared with usual care in patients with cardiovascular disease or at high risk: a prospective, individual patient data meta-analysis of 3140 patients in six countries. Int J Cardiol 2016;205:147-56.

21 Redfern J, Usherwood T, Harris MF, et al. A randomised controlled trial of a consumer-focused e-health strategy for cardiovascular risk management in primary care: the consumer navigation of electronic cardiovascular tools (connect) study protocol. BMJ Open 2014;4:e004523.

22 Debray TPA, Moons KGM, Abo-Zaid GMA, et al. Individual participant data meta-analysis for a binary outcome: one-stage or two-stage? PLoS One 2013;8:e60650.

23 Ramachandran A, Snehalatha C, Ram J, et al. Effectiveness of mobile phone messaging in prevention of type 2 diabetes by lifestyle modification in men in India: a prospective, parallelgroup, randomised controlled trial. Lancet Diabetes Endocrinol 2013;1:191-8.

24 Chow CK, Redfern J, Hillis GS, et al. Effect of Lifestyle-Focused text messaging on risk factor modification in patients with coronary heart disease: a randomized clinical trial. JAMA 2015;314:1255-63.

25 Pfaeffli Dale L, Whittaker R, Jiang Y, et al. Text message and Internet support for coronary heart disease self-management: results from the Text4Heart randomized controlled trial. J Med Internet Res 2015;17:e237.

26 Maddison R, Whittaker R, Stewart R, et al. Heart: heart exercise and remote technologies: a randomized controlled trial study protocol. BMC Cardiovasc Disord 2011;11:26.

27 Arora S, Peters AL, Burner E, et al. Trial to examine text messagebased mHealth in emergency department patients with diabetes (TEXT-MED): a randomized controlled trial. Ann Emerg Med 2014:63:745-54.

28 Shariful Islam SM, Niessen LW, Ferrari U, et al. Effects of mobile phone SMS to improve glycemic control among patients with type 2 diabetes in Bangladesh: a prospective, parallel-group, randomized controlled trial. Diabetes Care 2015;38:e112-3.

29 Wald DS, Bestwick JP, Raiman L, et al. Randomised trial of text messaging on adherence to cardiovascular preventive treatment (interact trial). PLoS One 2014;9:e114268.

30 Morisky DE, Ang A, Krousel-Wood M, et al. Predictive validity of a medication adherence measure in an outpatient setting. $J$ Clin Hypertens 2008;10:348-54.

31 Maddison R, Pfaeffli L, Whittaker R, et al. A mobile phone intervention increases physical activity in people with cardiovascular disease: results from the heart randomized controlled trial. Eur J Prev Cardiol 2015;22:701-9.

32 Shariful Islam SM, Lechner A, Ferrari U, et al. Mobile phone use and willingness to pay for SMS for diabetes in Bangladesh. J Public Health 2016;38:163-9.

33 Burn E, Nghiem S, Jan S, et al. Cost-Effectiveness of a text message programme for the prevention of recurrent cardiovascular events. Heart 2017;103:893-4.

34 Vargas G, Cajita MI, Whitehouse E, et al. Use of short messaging service for hypertension management: a systematic review. $J$ Cardiovasc Nurs 2017;32:260.

35 Rees K, Bennett P, West R, et al. Psychological interventions for coronary heart disease (cochrane review). Oxford: Update Software, 2004.

36 Clark AM, Hartling L, Vandermeer B, et al. Meta-analysis: secondary prevention programs for patients with coronary artery disease. Ann Intern Med 2005;143:659-72.

37 Artinian NT, Fletcher GF, Mozaffarian D, et al. Interventions to promote physical activity and dietary lifestyle changes for cardiovascular risk factor reduction in adults: a scientific statement from the American heart association. Circulation 2010;122:406-41.

38 Balady GJ, Williams MA, Ades PA, et al. Core components of cardiac rehabilitation/secondary prevention programs: 2007 update: a scientific statement from the American heart association exercise, cardiac rehabilitation, and prevention Committee, the Council on clinical cardiology; the councils on cardiovascular nursing, epidemiology and prevention, and nutrition, physical activity, and metabolism; and the American association of cardiovascular and pulmonary rehabilitation. Circulation 2007;115:2675-82.

39 Auer R, Gaume J, Rodondi N, et al. Efficacy of in-hospital multidimensional interventions of secondary prevention after acute coronary syndrome: a systematic review and meta-analysis. Circulation 2008;117:3109-17.

40 Burell G, Granlund B. Women's hearts need special treatment. Int J Behav Med 2002:9:228-42.

41 Chow CK, Redfern J, Thiagalingam A, et al. Design and rationale of the tobacco, exercise and diet messages (text me) trial of a text message-based intervention for ongoing prevention of cardiovascular disease in people with coronary disease: a randomised controlled trial protocol. BMJ Open 2012;2:e000606.

42 Bobrow K, Brennan T, Springer D, et al. Efficacy of a text messaging (SMS) based intervention for adults with hypertension: protocol for the StAR (SMS Text-message adherence suppoRt trial) randomised controlled trial. BMC Public Health 2014;14:28.

43 Islam SMS, Lechner A, Ferrari U, et al. Mobile phone intervention for increasing adherence to treatment for type 2 diabetes in an urban area of Bangladesh: protocol for a randomized controlled trial. BMC Health Serv Res 2014;14:586.

44 Dale LP, Whittaker R, Jiang Y, et al. Improving coronary heart disease self-management using mobile technologies (Text4Heart): a randomised controlled trial protocol. Trials 2014;15:71. 